\title{
Controlling the Flow of Light Using the Inhomogeneous Effective Gauge Field that Emerges from Dynamic Modulation
}

\author{
Kejie Fang* \\ Department of Physics, Stanford University, Stanford, California 94305, USA
}

Shanhui Fan

Department of Electrical Engineering, Stanford University, Stanford, California 94305, USA

(Received 29 April 2013; revised manuscript received 19 August 2013; published 11 November 2013)

We show that the effective gauge field for photons provides a versatile platform for controlling the flow of light. As an example we consider a photonic resonator lattice where the coupling strength between nearest neighbor resonators are harmonically modulated. By choosing different spatial distributions of the modulation phases, and hence imposing different inhomogeneous effective magnetic field configurations, we numerically demonstrate a wide variety of propagation effects including negative refraction, one-way mirror, and on- and off-axis focusing. Since the effective gauge field is imposed dynamically after a structure is constructed, our work points to the importance of the temporal degree of freedom for controlling the spatial flow of light.

DOI: 10.1103/PhysRevLett.111.203901

PACS numbers: 42.25.Gy, 42.79.Nv, 42.82.Et

It was recently recognized that when a photonic structure undergoes dynamic refractive index modulation, the phase of the modulation creates an effective gauge potential and effective magnetic field for photons [1-3]. The effective magnetic field can induce photonic phenomena similar to charged particles under a real magnetic field, such as a photonic one-way edge mode [2] and a photonic de Haasvan Alphen effect [4]. In this Letter, we further show that the use of inhomogeneous effective gauge fields provides additional degrees of freedom in controlling the flow of light. As examples, we show that one can achieve negative refraction, one-way mirrors, circulators, and focusing, based on the same resonator lattice structure subject to different configurations of inhomogeneous effective gauge fields.

Tailoring the propagation of light has been a central goal of nanophotonic research, which is critical for applications in on-chip communications and information processing [5]. Examples of previous studies include the use of waveguide arrays [6,7], photonic crystals [8-11], and metamaterials [12-14] to achieve various beam propagation effects within these structures. Moreover, by introducing inhomogeneity into these structures $[15,16]$, one can realize photon flow that emulates electron motion under an electric field [17-19].

Complementary to these works, which have largely focused on spatial degrees of freedom, our results here show that temporal degrees of freedom in a dynamic structure can also be quite useful in the control of electromagnetic wave propagations in space. Unlike the spatial (i.e., the structural) degrees of freedom, which are mostly defined by fabrication processes, the modulation phases can be readily changed in the dynamic structure, after the structure is constructed. Moreover, nonreciprocity, or timereversal symmetry breaking, which is difficult to achieve in static structures unless magneto-optical materials are used, arises rather naturally in dynamically modulated structures. Therefore, our approach of using a photonic gauge field provides additional degrees of flexibility in controlling light propagation.

To illustrate the idea we use the model system introduced in Ref. [2] that consists of a two-dimensional photonic resonator lattice as shown in Fig. 1. The lattice has a square unit cell and each unit cell contains two resonators $A$ and $B$ with different resonant frequencies $\omega_{A}$ and $\omega_{B}$, respectively. We assume only nearest-neighbor coupling with a form of $V \cos (\Omega t+\phi)$, where $V$ is the coupling strength, $\Omega$ and $\phi$ are the frequency and phase of the modulation. The Hamiltonian $H(t)$ of this resonator lattice is

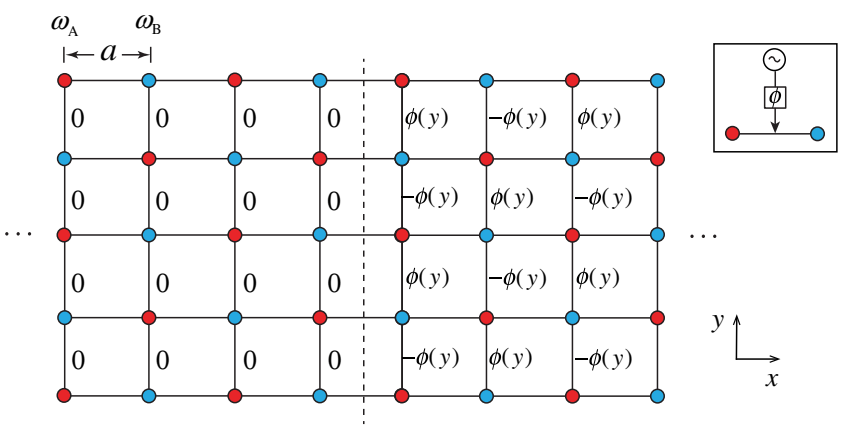

FIG. 1 (color online). A resonator lattice consisting of two kinds of resonators (red and blue dots) with dynamically modulated nearest neighbor coupling. The lattice is divided into two regions: the left region has an effective gauge potential $A_{y}=0$ and the right region has an effective gauge potential $A_{y}=$ $\phi(y) / a$. Inset: the phase of the modulated coupling between nearest neighbor resonators corresponds to the rf wave that generates the modulation, and can be externally set by $\mathrm{rf}$ generators or rf phase shifters. 


$$
\begin{aligned}
H(t)= & \omega_{A} \sum_{i} a_{i}^{\dagger} a_{i}+\omega_{B} \sum_{j} b_{j}^{\dagger} b_{j}+\sum_{\langle i j\rangle} V \cos \left(\Omega t+\phi_{i j}\right) \\
& \times\left(a_{i}^{\dagger} b_{j}+b_{j}^{\dagger} a_{i}\right)
\end{aligned}
$$

where $a_{i}^{\dagger}\left(a_{i}\right)$ and $b_{j}^{\dagger}\left(b_{j}\right)$ are the creation (annihilation) operators of the $A$ and $B$ resonators, respectively, and $\phi_{i j}$ is the phase of the modulation between resonators at nearest neighbor sites $i$ and $j$.

We will assume an on-resonance modulation, i.e., $\Omega=$ $\left|\omega_{A}-\omega_{B}\right|$, and stay in the regime $V \ll \Omega$ such that the rotation wave approximation applies. In such a case, when $\phi_{i j}=0$ everywhere, the structure is periodic and is described by a Floquet band structure [4], $\epsilon\left(k_{x}, k_{y}\right)=$ $V\left[\cos \left(a k_{x}\right)+\cos \left(a k_{y}\right)\right]$, where $\epsilon$ is the quasienergy, $a$ is the separation between two nearest neighbor resonators, and $\vec{k}=k_{x} \hat{e}_{x}+k_{y} \hat{e}_{y}$ is the Bloch wave vector, which has no direct and simple relation to the free space wavelength or wave vector of light.

In this system as described by Eq. (1), an effective gauge field arises from the spatial distribution of the modulation phase $\phi_{i j}$. This can be seen by going to a rotating frame, in which case the modulation phases then appear as the phases of the coupling constants in a time-independent tightbinding model, which gives rise to a gauge field structure through the Peierls substitution [2]. The value of the effective gauge field along the bond between sites $i$ and $j$ is determined by $\vec{A}=\vec{l}_{i j} \phi_{i j} / a$ [1], where $\vec{l}_{i j}$ is a unit vector that points from site $i$ in the $A$ sublattice to site $j$ in the $B$ sublattice. Furthermore, a nonuniform $\phi_{i j}$ distribution can create an effective magnetic field. The effective magnetic flux through a plaquette is defined as $B_{\text {eff }}=\oint \vec{A} \cdot d \vec{l} / a^{2}$ [2], where the integration is along the sides of a plaquette. The consequences of having a uniform magnetic field in the lattice have been explored in Refs. [2,4].

In this Letter, we study the $\phi_{i j}$ distribution as shown in Fig. 1, which corresponds to a nonuniform magnetic field, as we will see later. The lattice in Fig. 1 can be separated into left and right regions. In the left region, the phases on the bonds along both $x$ and $y$ axes are all zero. Therefore, the left region has a zero effective gauge potential and a zero effective magnetic field. We excite a beam by placing a source in the left region. In the right region, the phases on the bonds along the $x$ axis are zero, but the phases on the bonds along the $y$ axis are a function $\phi(y)$ of $y$ and alternate between positive and negative values. The different spatial configurations of $\phi(y)$ then correspond to different configurations of effective gauge potential and effective magnetic field in the right region. By choosing a different modulation phase distribution in the right region, i.e., by choosing different $\phi(y)$, we can achieve versatile control of light propagation effects, for the beam incident from the left.

To simulate the motion of light in the presence of a source in this dynamically modulated resonator lattice we solve the coupled-mode equation [4],

$$
i \frac{d|\psi\rangle}{d t}=H(t)|\psi\rangle+|s\rangle,
$$

where $|\psi\rangle=\left[\sum_{i} v_{i}(t) a_{i}^{\dagger}+\sum_{j} v_{j}(t) b_{j}^{\dagger}\right]|0\rangle$ is the photon state with $v_{i(j)}(t)$ being the amplitude at site $i(j)$. In our simulations, we use a source $|s\rangle$ that takes the form

$$
|s\rangle=\sum_{x, y} e^{-\left[\left(x-x_{0}\right)^{2}+\left(y-y_{0}\right)^{2}\right] / w^{2}} e^{i\left(k_{x 0} x+k_{y 0} y\right)-i\left[\omega_{x, y}+\epsilon\left(k_{x 0}, k_{y 0}\right)\right] t} a^{\dagger}\left(b^{\dagger}\right)_{\{x, y\}}|0\rangle,
$$

in order to create a beam with a spatial Gaussian profile. In (3), $w$ is the width of the beam, $\left\{x_{0}, y_{0}\right\}$ is the center of the source, $\left\{k_{x 0}, k_{y 0}\right\}$ are the Bloch momentum of the beam, $\omega_{x, y}$ is the frequency of the resonator at coordinate $\{x, y\}$.

We first study the special case where in the right region $\phi(y)$ is a constant, i.e., $\phi(y) \equiv \phi$. In this case, both the left and the right regions have a zero effective magnetic field. Nevertheless, we demonstrate that a beam propagating across the interface between these two regions undergoes refraction, and under a certain condition, even negative refraction can happen. To understand such an effect, we investigate the band structures for the two regions of Fig. 1 . For the left and right regions, the Floquet band structures are given by $\epsilon\left(k_{x}, k_{y}\right)=V\left[\cos \left(a k_{x}\right)+\cos \left(a k_{y}\right)\right]$ and $\epsilon\left(k_{x}, k_{y}\right)=V\left[\cos \left(a k_{x}\right)+\cos \left(a k_{y}-\phi\right)\right], \quad$ respectively. Thus, in the momentum space, the band structure of the right region is shifted along the $y$ direction by $\Delta k_{y}=\phi / a$, as compared to the band structure of the left region. In general, arbitrary shift of the band structure in momentum space can be accomplished by the appropriate choice of phase distribution. Such a capability for achieving a shift of the photonic band structure in momentum space is quite unique in our dynamically modulated systems, and has not been noted in any other system before.

As a beam propagates through the interface between the left and right regions, the relation between the incident angle $\theta_{i}$ and refraction angle $\theta_{r}$ of a beam can be obtained by considering the conservation of quasienergy and surface-parallel momentum. Suppose the momentum of the beam is small and thus the band structure in the left region can be approximated by $\epsilon(\vec{k}) \approx 2 V-V(a k)^{2}$. The constant quasienergy contour is a circle centered around $\vec{k}=0$. At the same quasienergy, the constant quasienergy contour in the right region is shifted by $\phi / a$ along the $y$ axis as shown in Fig. 2(a). Therefore, the relation between $\theta_{i}$ and $\theta_{r}$ becomes 
(a)

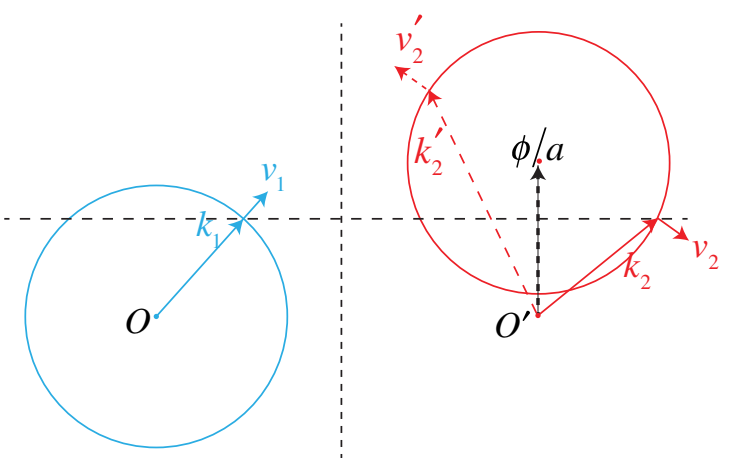

(b)

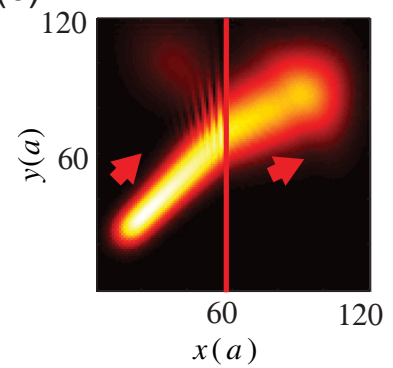

(c)

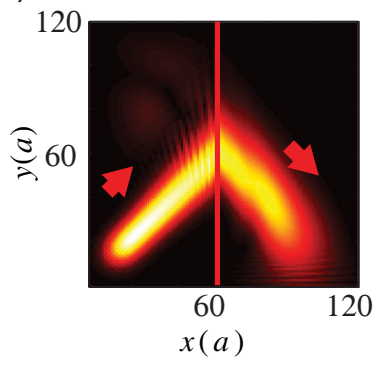

FIG. 2 (color online). (a) Analysis of beam refraction for the structure in Fig. 1, for $\phi(y) \equiv \phi$. Circles are the constant quasienergy contours in the left and right regions, respectively. $k_{1,2}$ and $v_{1,2}$ are the incident and refracted momentum and group velocity of the beam. $k_{1}$ and $k_{2}$ have equal parallel components. $k_{2}^{\prime}$ and $v_{2}^{\prime}$ are the time reversal of the refracted beam that corresponds to $k_{2}$. (b) Simulation for the case of $\phi=0.05$ showing positive refraction. (c) Simulation for the case of $\phi=2$ demonstrating negative refraction.

$$
\sin \theta_{i}-\sin \theta_{r}=\frac{\phi}{a\left|\vec{k}_{0}\right|},
$$

where $\vec{k}_{0}$ is the Bloch momentum of the incident beam [20]. From Eq. (4), we see that $\theta_{r}<0$, and hence negative refraction occurs, when $\phi /(2 a)<\left|\vec{k}_{0}\right|$ and $\max \left\{\sin ^{-1}\left[\phi /\left(a\left|\vec{k}_{0}\right|\right)-1\right], 0\right\}<\theta_{i}<\min \left\{\sin ^{-1}\left[\phi /\left(a\left|\vec{k}_{0}\right|\right)\right]\right.$, $\pi / 2\}$.

We verify the above analytical theory with direct numerical calculation using Eq. (2). We take $\theta_{i}=45^{\circ}$, $\left|\vec{k}_{0}\right|=1 / a$. In Fig. 2(b), we assume $\phi=0.05$ in the right region. In this case, the shift in the momentum space for the constant quasienergy contour is small, and the beam passes through the interface with a small angle of refraction. In Fig. 2(c), we assume $\phi=2$, which generates a larger shift of the constant quasienergy contour in the right region, and thus the beam undergoes negative refraction as it passes through the interface. Also, in both cases of Figs. 2(b) and 2(c), we observe almost no reflection at the interface. This is due to the impedance matching between the two regions, since aside from the phases of the coupling constants all other parameters of the Hamiltonian are the same on both sides.

(a)
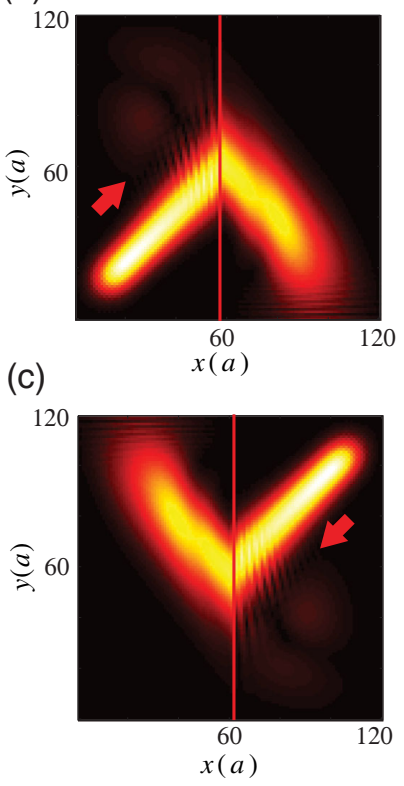

(d)
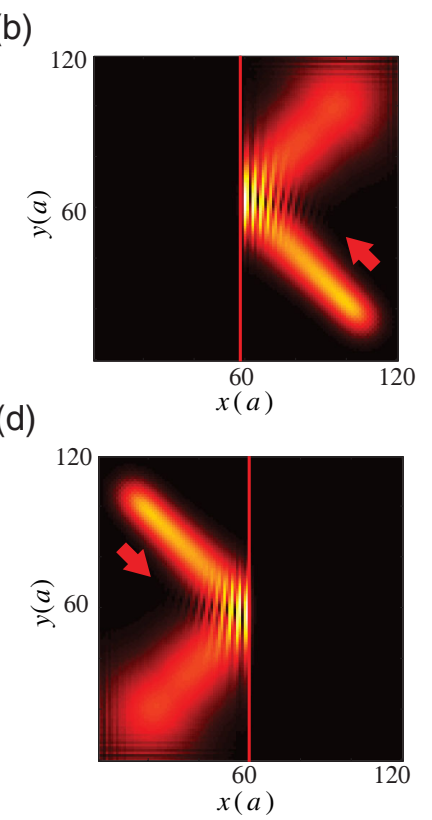

FIG. 3 (color online). Demonstration of the interface of the two regions in Fig. 1 functioning as a circulator. The right region has $\phi=2$. Arrows indicate the incident direction of the beams.

The refraction of beam in this lattice may seem counterintuitive, since the left and right regions have zero effective magnetic fields. Scrutinizing the structure of Fig. 1 reveals that there are nonvanishing effective magnetic fields located at the interface of the two regions, since the phase accumulation around the plaquettes on the interface is nonzero. Thus, as an alternative to the previous explanation using the shift of band structure, one can equivalently state that the magnetic fields at the interface supply a canonical momentum (not the conserved Bloch momentum $k_{y}$ ) kick to the incident beam, leading to refraction. Such a kind of magnetic flux induced beam refraction is difficult to observe for electrons, since one needs to achieve a magnetic field sheet with the density of the magnetic flux quantum.

In our structure, the momentum kick from the effective magnetic field at the interface is reminiscent of the concept of metasurfaces [21,22], or the nonlinear processes that give rise to negative refraction [23]. However, unlike the metasurfaces in Refs. [21,22], here the effective magnetic field breaks time-reversal symmetry. As a result, the beam propagation is nonreciprocal. As an illustration, we start with Fig. 3(a), which reproduces Fig. 2(b) where a beam incident from the left region undergoes negative refraction as it passes through the interface. In Fig. 3(b), we excite a beam, incident upon the interface from the right region, propagating along the direction opposite to the outgoing beam in Fig. 3(a). We observe instead total internal reflection at the interface. Such a total internal reflection can be accounted for by also examining Fig. 2(a), where we note that in this case the incident beam from the right region 
[dashed arrows in Fig. 2(a)] cannot excite any mode in the left region by momentum conservation consideration. Such a one-way total internal reflection has been previously considered in magneto-optical photonic crystals [24]. Here we achieve a similar effect without the use of magneto-optics.

Based on such a one-way total internal reflection effect, the interface between the left and right regions in fact functions as a four-port circulator, where the ports corresponding to the four incident beam directions are as shown in the four panels of Fig. 3. Circulators have been previously considered for a guided mode using either the magneto-optical effect $[25,26]$ or with the use of dynamic modulation [27]. Here we show that a single interface can behave as a circulator for beams without guiding structure.

The previous case corresponds to a constant $\phi(y)$ in the right region of the lattice. Next we consider the case where $\phi(y)$ is not a constant, which provides additional capabilities for beam manipulation. As a specific example, we design $\phi(y)$ to realize both on-axis and off-axis focusing effects for a collimated beam propagating along the $x$ axis in the left region and normally incident onto the interface. We assume that the center of the incident beam is located at $y=0$. To design $\phi(y)$ we follow a ray tracing procedure. For a ray incident upon a position $y$ at the interface, we choose $\phi(y)$ according to Eq. (4) to achieve an angle of refraction that is appropriate for focusing. To create a focal point located at $(f, d)$ to the right of the interface, the ray tracing procedure above results in

$$
\phi(y)=a k_{0} \frac{y-d}{\sqrt{(y-d)^{2}+f^{2}}} .
$$

In Fig. 4, using Eq. (5), we generate two different modulation phase distributions. Figure 4(a) uses the parameters $f=60 a$ and $d=0 a$, and Fig. 4(b) uses the parameters $f=60 a$ and $d=20 a$. From the ray tracing procedure above, we expect on-axis focusing with respect to the beam axis in Fig. 4(a), and off-axis focusing in Fig. 4(b), which is indeed what we observe in the numerical simulations of Fig. 4. Again, we emphasize that the two cases in Fig. 4 represent exactly the same structure, except with different modulation phase distributions, indicating versatile reconfigurability that is inherent in the use of such an effective gauge field.

Given the agreement between our analytic descriptions of the effect of the gauge fields, and the numerical simulations in two dimensions, below we will use the analytic description to design 3D structures. We extend the lattice of Fig. 1 in the $z$ direction to make a cubic lattice. The two kind of resonators are alternatively distributed in the cubic lattice with dynamically modulated nearest neighbor coupling. We separate the lattice into two regions. In the left region $(x<0)$, all the modulation phases on the bonds along the three axes are zero; in the right region $(x>0)$, the phases on the bonds along the $x$ axis are all zero, but the
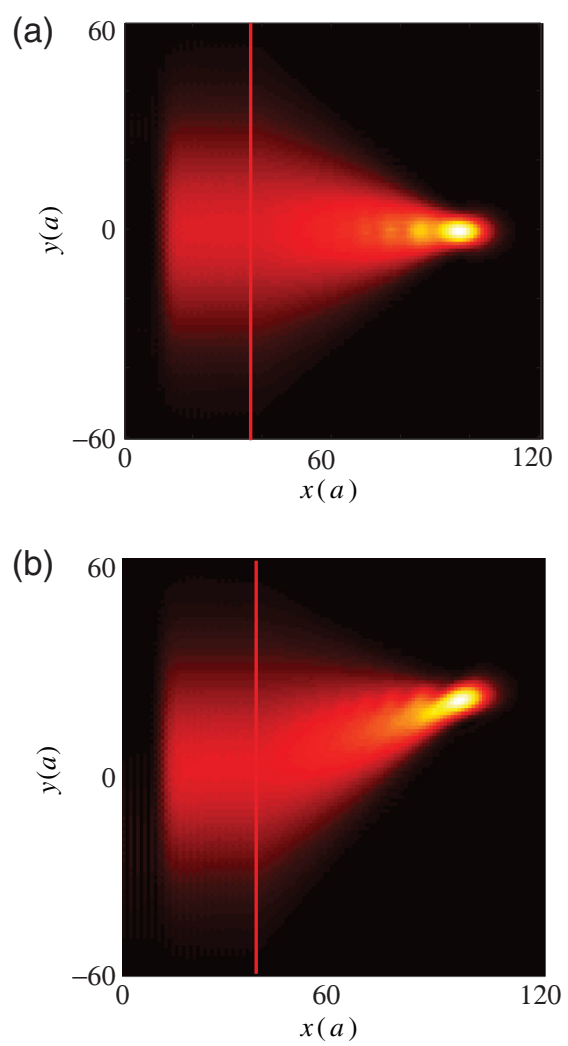

FIG. 4 (color online). A collimated beam is focused on axis (a) and off axis (b) by designing $\phi(y)$ according to Eq. (5) in the right region of Fig. 1.

phases along the $y$ axis $\left[\phi_{y}(y, z)\right]$ and along the $z$ axis $\left[\phi_{z}(y, z)\right]$ are functions of $y$ and $z$. To achieve a positiondependent refraction, the beam refraction across the interface at $x=0$ can be determined by the following equations:

$$
\begin{aligned}
& \phi_{y}(y, z)=a k_{0}\left(\sin \theta_{i y}-\sin \theta_{r y}\right), \\
& \phi_{z}(y, z)=a k_{0}\left(\sin \theta_{i z}-\sin \theta_{r z}\right),
\end{aligned}
$$

where $\theta_{i(r) y, z}$ are the directional angle of incident (refracted) beam along $y$ and $z$ axes respectively. Thus, the desired functionality, such as focusing, which is described by the $\theta$ 's, can then be implemented in our lattice through Eqs. (6) and (7).

As final remarks, the experimental implementation of the Hamiltonian of Eq. (1) has been considered in Ref. [2], and the key arguments are reproduced in the Supplemental Material [28]. The dynamically modulated coupling between the two resonances, which is the crucial aspect that enables the creation of an effective gauge field, can be achieved using a mixer in the microwave frequency range, or a modulator in the optical frequency range. In the microwave frequency range, using such a mixer to create a gauge potential has already been demonstrated experimentally in Ref. [3]. In the experiment of Ref. [3], the modulation phase is the phase of the local oscillator, which 
can be arbitrarily set after the structure is constructed. In the optical frequency range, the weak modulation strength $(\Delta n / n)$ leads to a relative weak coupling between resonators; however, the modulation phase again can be arbitrarily set by external modulation sources, as demonstrated in a recent experiment integrating silicon modulators [29], and thus the amplitude of the gauge field is not limited. Such a dynamic aspect of the gauge field, as well as the nonreciprocity generated by such a gauge field, differs significantly from several recent proposals and experiments that create an effective gauge field based on a spin degree of freedom in photons [30-33]. We also note the effects shown in the Letter are robust to a certain amount of resonant frequency disorders for practical considerations (see Supplemental Material [28]). In conclusion, our work indicates significant new capabilities for controlling the spatial flow of light, through the control of temporal degrees of freedom that generate an effective gauge field for photons.

This work is supported in part by U.S. Air Force Office of Scientific Research Grant No. FA9550-09-1-0704, and U.S. National Science Foundation Grant No. ECCS-1201914.

*Present address: Thomas J. Watson, Sr., Laboratory of Applied Physics, California Institute of Technology, Pasadena, CA 91125, USA.

[1] K. Fang, Z. Yu, and S. Fan, Phys. Rev. Lett. 108, 153901 (2012).

[2] K. Fang, Z. Yu, and S. Fan, Nat. Photonics 6, 782 (2012).

[3] K. Fang, Z. Yu, and S. Fan, Phys. Rev. B 87, 060301(R) (2013).

[4] K. Fang, Z. Yu, and S. Fan, Opt. Express 21, 18216 (2013).

[5] J. D. Joannopoulos et al., Photonic Crystal-Molding the Flow of Light (Princeton University Press, Princeton, NJ, 2008).

[6] D. N. Christodoulides, F. Lederer, and Y. Silberberg, Nature (London) 424, 817 (2003).

[7] L. Verslegers, P. B. Catrysse, Z. Yu, and S. Fan, Phys. Rev. Lett. 103, 033902 (2009).

[8] C. Luo, S. G. Johnson, J. D. Joannopoulos, and J.B. Pendry, Phys. Rev. B 68, 045115 (2003).

[9] E. Cubukcu, K. Aydin, E. Ozbay, S. Foteinopoulou, and C. Soukoulis, Phys. Rev. Lett. 91, 207401 (2003).

[10] L. Wu, M. Mazilu, and T. F. Krauss, J. Lightwave Technol. 21, 561 (2003).
[11] Y.A. Urzhumov and D. R. Smith, Phys. Rev. Lett. 105, 163901 (2010).

[12] A. Ishikawa, S. Zhang, D. Genov, G. Bartal, and X. Zhang, Phys. Rev. Lett. 102, 043904 (2009).

[13] F. Lemoult, N. Kaina, M. Fink, and G. Lerosey, Nat. Phys. 9, 55 (2013)

[14] J. Quach, C. Su, and A. Greentree, Opt. Express 21, 5575 (2013).

[15] P. St. J. Russell and T. A. Birks, J. Lightwave Technol. 17, 1982 (1999)

[16] Y. Jiao, S. Fan, and D. A. B. Miller, Phys. Rev. E 70, 036612 (2004).

[17] R. Morandotti, U. Peschel, J. Aitchison, H. Eisenberg, and Y. Silberberg, Phys. Rev. Lett. 83, 4756 (1999).

[18] R. Khomeriki and S. Ruffo, Phys. Rev. Lett. 94, 113904 (2005).

[19] H. Trompeter, W. Krolikowski, D. Neshev, A. Desyatnikov, A. Sukhorukov, Y. Kivshar, T. Pertsch, U. Peschel, and F. Lederer, Phys. Rev. Lett. 96, 053903 (2006).

[20] For larger beam momentum, Eq. (4) should be modified using the exact constant quasienergy contour determined by $\cos \left(a k_{x}\right)+\cos \left(a k_{y}\right)=$ const. However, similar beam control can still be achieved.

[21] N. Yu, P. Genevet, M. A. Kats, F. Aieta, J.-P. Tetienne, F. Capasso, and Z. Gaburro, Science 334, 333 (2011).

[22] A. V. Kildishev, A. Boltasseva, and V. M. Shalaev, Science 339, 1232009 (2013).

[23] H. Harutyunyan, R. Beams, and L. Novotny, Nat. Phys. 9, 423 (2013).

[24] Z. Yu, Z. Wang, and S. Fan, Appl. Phys. Lett. 90, 121133 (2007).

[25] D. M. Pozar, Microwave Engineering (Wiley, New York, 2011), Chap. 9, p. 487.

[26] Z. Wang and S. Fan, Opt. Lett. 30, 1989 (2005).

[27] Z. Yu and S. Fan, Appl. Phys. Lett. 94, 171116 (2009).

[28] See Supplemental Material at http://link.aps.org/ supplemental/10.1103/PhysRevLett.111.203901 for experimental implementation and the effect of disorder.

[29] L. Tzuang, K. Fang, S. Fan, and M. Lipson, arXiv:1309.5269.

[30] M. Hafezi, E. A. Demler, M. D. Lukin, and J. M. Taylor, Nat. Phys. 7, 907 (2011).

[31] R. O. Umucalilar and I. Carusotto, Phys. Rev. A 84, 043804 (2011).

[32] A. B. Khanikaev, S. H. Mousavi, W.-K. Tse, M. Kargarian, A. H. MacDonald, and G. Shvets, Nat. Mater. 12, 233 (2013).

[33] M. C. Rechtsman, J. M. Zeuner, Y. Plotnik, Y. Lumer, D. Podolsky, F. Dreisow, S. Nolte, M. Segev, and A. Szameit, Nature (London) 496, 196 (2013). 